\title{
Predictive Maintenance Plan for Switched Reluctance Motor Drives
}

\author{
J. A. Sánchez, P. Andrada, B. Blanqué, M. Torrent
}

\begin{abstract}
In a near future when Industry 4.0 is implanted, predictive maintenance will have a much more prominent role than that currently plays in traditional industry. Predictive maintenance is the organized monitoring with periodic or continuous measurement of variables of the state of the equipment to be maintained and its comparison with preestablished patterns for the determination of the instant in which the maintenance intervention should take place. Three-phase induction motor drives are one of the industrial equipment that has been given greater attention regarding predictive maintenance plans. In recent years, switched reluctance motor drives have begun to have some relevance in the industry. In this paper, after a brief review of the switched reluctance motors and their control, an analysis of the different types of faults and a description of the most usual diagnostic techniques in electric drives, a predictive maintenance plan for switched reluctance motor drives is proposed. This predictive maintenance plan considers different control strategies (hysteresis and single pulse), and is based on diagnostic techniques such as analysis of currents and spectral analysis of vibrations. Exhaustive experimental tests have been carried out in order to validate the proposed plan.
\end{abstract}

Index Terms - diagnostic techniques, faults, predictive maintenance, switched reluctance motor.

\section{INTRODUCCION}

$\mathrm{H}^{\mathrm{r}}$ OY por hoy, la industria se enfrenta a una nueva manera de organizar la producción, Industria 4.0, que tiene como finalidad crear fábricas inteligentes que mejoren la adaptabilidad de los medios de producción, no sólo para producir más y mejor con menores costes, sino también para poder atender a las peticiones personalizadas de los clientes. La industria 4.0 se basa en nuevas tecnologías como: inteligencia artificial, internet de las cosas, sistemas ciberfísicos, big data, realidad virtual y aumentada, robótica avanzada y fabricación aditiva [1]. En este nuevo entorno industrial el mantenimiento predictivo tendrá un papel mucho más destacado que el que juega, actualmente, en la industria tradicional $[2,3]$.

El mantenimiento predictivo es el seguimiento organizado con medición periódica o continua de variables del estado de

Los autores forman parte del Grupo de Accionamientos Eléctricos con Conmutación Electrónica (GAECE), Departamento de Ingenieria Eléctrica (DEE). Escola Politècnica Superior d'Enginyeria de Vilanova i la Geltrú (EPSEVG). Universitat Politècnica de Catalunya (UPC), BARCELONATECH. 08800 Vilanova i la Geltrú, Barcelona, Spain (e-mail: jsanchez@ee.upc.edu). los equipos a mantener y su comparación con unos patrones preestablecidos para la determinación del instante en que se debe producir la intervención de mantenimiento [4].

En la industria, los accionamientos con motores eléctricos rotativos $\mathrm{y}$ en especial los accionamientos con motores asíncronos trifásicos son uno de los equipos industriales a los que mayor atención se ha prestado en lo concerniente a planes de mantenimiento predictivo basados en la investigación en técnicas de diagnóstico para la detección de las faltas en este tipo de accionamiento [5, 6].

En los últimos años los accionamientos con motores de reluctancia autoconmutados (SRM) empiezan a tener cierta relevancia en el ámbito industrial, en este sentido es significativo señalar que el mercado de accionamientos eléctricos con SRM en los últimos cuatro años ha mantenido un crecimiento anual del 4.73\%, pasando de 370, en 2013, a 425 millones de USD en 2016, y esperando alcanzar los 510 millones de USD en 2021 [7].

Atendiendo a este crecimiento y a la que parece imparable implantación de la Industria 4.0, en este artículo se propone un plan de mantenimiento predictivo específico para los accionamientos con SRM. Este plan de mantenimiento predictivo se basa en la selección de las técnicas de diagnóstico más adecuadas, entre las habituales en máquinas eléctricas rotativas, para cada tipo de falta y según el control aplicado. Después de esta introducción, el artículo se organiza de la siguiente manera: en la sección II se describen las distintas estrategias de control y los tipos de faltas en los motores de reluctancia autoconmutados; en la sección III se enumeran las técnicas de diagnóstico utilizadas en el mantenimiento predictivo; en la sección IV se presenta un plan de mantenimiento predictivo para los accionamientos con motores de reluctancia autoconmutados basado en pruebas experimentales; finalmente en la sección $\mathrm{V}$ se exponen las conclusiones que se derivan del trabajo realizado.

\section{Motores De Reluctancia Autoconmutados}

\section{A. Control de accionamientos con motores de reluctancia} autoconmutados.

La máquina de reluctancia autoconmutada es un convertidor electromecánico de la energía de simple excitación que normalmente funciona fuertemente saturado, con polos salientes en el estator y en el rotor, y bobinas concentradas enrolladas en los polos estatóricos que conectadas adecuadamente forman las fases de la máquina. El par se produce por la tendencia del rotor a moverse hacia una 
posición en la que se maximice la inductancia del devanado de fase excitado, es decir, para conseguir el alineamiento de los polos del estator con los del rotor. Para ello, es necesario un convertidor de potencia, con interruptores de estado sólido, que generen la correcta secuencia de conmutación de las fases para lo cual es preciso conocer la posición del rotor, circunstancia que requiere de un sensor de posición.

En los SRM se puede variar la velocidad actuando:

- sobre la tensión aplicada en las fases, V, modificando la relación cíclica $\mathrm{D}$, según la ecuación (1):

$\mathrm{D}=\frac{\mathrm{T}_{\mathrm{ON}}}{\mathrm{T}}$

Dónde:

$\mathrm{T}_{\mathrm{ON}}=$ tiempo de conducción.

$\mathrm{T}$ = periodo de conducción.

- sobre la corriente (modificando la consigna o referencia de corriente).

- sobre el período de conducción, modificando el ángulo de inicio de conducción $\theta_{0}$, el ángulo de cese de conducción $\theta \mathrm{c}, \mathrm{o}$ ambos a la vez.

La característica par-velocidad natural de un SRM, manteniendo la tensión y el período de conducción constantes, es, idealmente, inversamente proporcional al cuadrado de la velocidad. Sin embargo, un SRM puede trabajar a potencia constante, par inversamente proporcional a la velocidad, manteniendo la tensión fija y variando el período de conducción. También es posible trabajar a par constante, a bajas velocidades, manteniendo constante el periodo de conducción y controlando la tensión o la corriente en las fases del motor. En los dos primeros casos el control es por pulso único y en el caso de par constante si el control es por tensión, control PWM, y si es por corriente, control por histéresis. En la Fig. 1 se muestra la forma de la corriente de fase de un SRM al pasar de control por histéresis a control de pulso único.

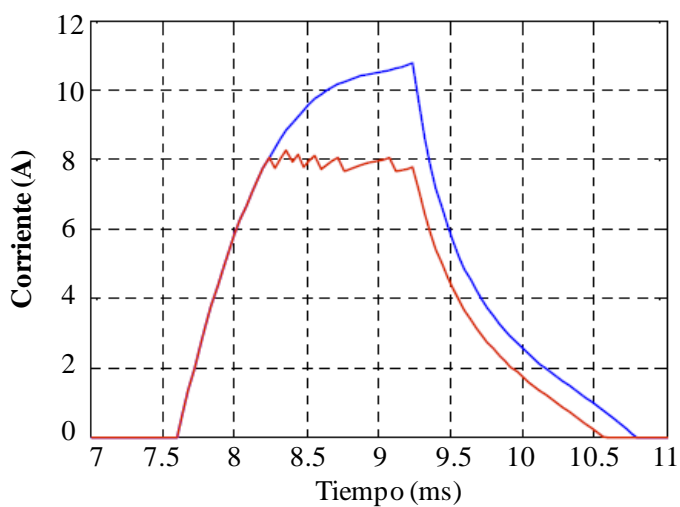

Fig. 1. Corriente de fase en un SRM con control de histéresis (rojo) y con control de pulso único (azul).

En la Fig. 2 se han subrayado sobre la característica parvelocidad las zonas con distintos rangos de velocidades asociadas con los tipos de operación antes mencionados. En la Tabla I se presenta de forma resumida los tipos o estrategias de control a utilizar para cada zona de funcionamiento indicando, en cada caso, las magnitudes que son variables y las que son constantes. Es importante señalar que dado que el par en un SRM puede expresarse como una función no lineal de múltiples variables, existen posibilidades de control más sofisticadas que las hasta ahora planteadas, como por ejemplo el control de tensión maximizando el rendimiento, lo que requiere que además de actuar sobre la relación cíclica se han de elegir los ángulos $\theta_{0}$ y $\theta_{\mathrm{c}}$ adecuados en cada instante.

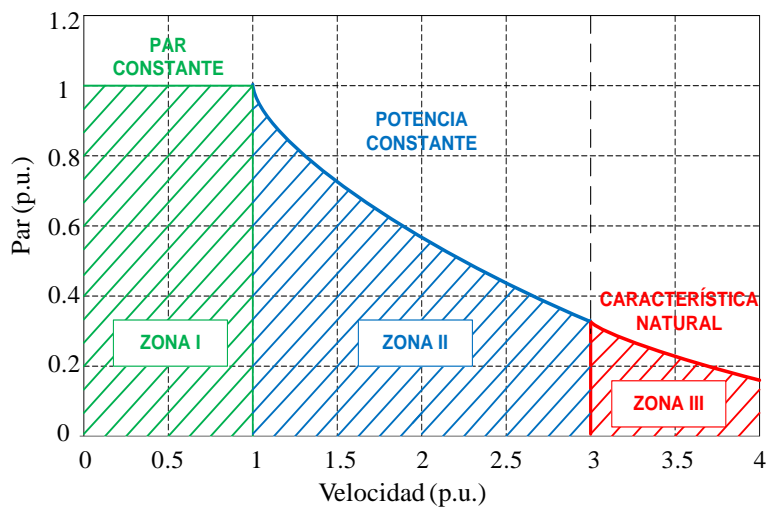

Fig. 2. Zonas de la característica par-velocidad asociadas a los distintos tipos de operación de un accionamiento SRM.

TABLA I

ESTRATEGIAS DE CONTROL EN CADA ZONA DE FUNCIONAMIENTO

\begin{tabular}{ccccc}
\hline \hline & Tipo de control & Operación & Variables & Constantes \\
\hline Zona I & Histéresis & Par constante & Iref & \\
& PWM & $\mathrm{D}$ & $\theta_{0} \mathrm{y} \theta_{\mathrm{c}}$ \\
Zona II & Pulso único & $\begin{array}{c}\text { Potencia } \\
\text { constante }\end{array}$ & $\theta_{0} \mathrm{y} \theta_{\mathrm{c}}$ & $\mathrm{V}$ \\
Zona III & Pulso único & $\begin{array}{c}\text { Característica } \\
\text { natural }\end{array}$ & $\theta_{0} \mathrm{y} \theta_{\mathrm{c}}$ \\
\hline \hline
\end{tabular}

\section{B. Análisis de faltas}

La aparición de una falta, cualquier variación accidental de una variable que ocasiona un mal funcionamiento de una parte o de todo un sistema, en un accionamiento puede tener serias repercusiones para el funcionamiento de la carga que arrastra. En la industria una falta se traduce, por lo general, en la interrupción del proceso y en la pérdida de la producción. En otros sectores, como el sector aeronáutico y aeroespacial, puede incluso poner en peligro la seguridad de las personas.

En las aplicaciones especialmente sensibles a faltas, es conveniente utilizar accionamientos tales que cuando aparezca alguna falta sean capaces de proporcionar prestaciones similares a las habituales (funcionamiento sin faltas). A un accionamiento de estas características se le denomina tolerante a faltas. Los SRM se ajustan bastante bien a los requisitos de un accionamiento tolerante a faltas debido a la ausencia de bobinados de excitación o de imanes permanentes en el rotor y a la independencia de las fases del motor. Además, las ramas del convertidor electrónico de potencia son así mismo independientes y prácticamente inmunes a disparos intempestivos. Por otro lado, una vez aparecida una falta ésta puede detectarse y aislarse de la fase o la rama afectada. En 
estas circunstancias, el SRM puede continuar en funcionamiento en condiciones satisfactorias, esto es sin efectos excesivos en lo referente a: oscilaciones de velocidad, de par, de vibraciones, de ruido, de incremento de temperatura o de reducción de potencia [8, 9].

En los SRM distinguiremos dos tipos de faltas:

- Faltas mecánicas.

- Faltas eléctricas.

Las faltas mecánicas son debidas a deficiencias constructivas como la excentricidad del rotor o desalineamiento en el acoplamiento del SRM con la máquina a accionar.

En las faltas eléctricas, podemos distinguir las faltas que provengan de la estructura magnética reluctante (propiamente el motor), del convertidor electrónico de potencia o de los captadores de posición, aunque algunas de ellas produzcan los mismos efectos. Entre las faltas en el motor hay que considerar: falta de fase a masa, falta de fase abierta, falta debida al cortocircuito total de una fase, falta debida al cortocircuito entre espiras de una bobina de una fase $y$ cortocircuito entre fases. Las faltas en el convertidor electrónico de potencia dependerán del tipo de convertidor elegido y las más importantes para el convertidor en puente asimétrico o clásico son: interruptor de una fase abierto, interruptor de una fase en cortocircuito y diodo de una fase abierto. Las faltas en los sensores de posición también dependen del tipo de sensor de posición a utilizar: optointerruptores asociados a un disco ranurado, encoder o resolver. Es importante añadir que cualquier perturbación en el sistema de control provocará un comportamiento incorrecto en el funcionamiento del motor que se verá reflejada, por lo general, en una forma anómala de la corriente. Los posibles fallos estarán condicionados por el control elegido y sus consecuencias se reflejarán por lo general en faltas en el convertidor estático.

\section{TÉCNICAS De DiAgNÓstiCO}

Las máquinas eléctricas en general son susceptibles de presentar distintos tipos de faltas. En la actualidad, en los sistemas productivos su diagnóstico precoz se traduce en una importante reducción de costes que justifican el uso de técnicas sofisticadas y de equipos caros para su detección. Existen distintas técnicas para la detección y diagnóstico de faltas, que podemos clasificar en dos tipos:

- Las que se llevan a cabo con la máquina parada (off line).

- Las que se realizan con la máquina en marcha (on line).

\section{A. Técnicas de diagnóstico de faltas off line}

Tienen el inconveniente de que han de realizarse con la máquina parada, por lo general se espera a realizarlos en los periodos de parada programada. En esta categoría se encuentran los ensayos de medidas de aislamiento para la comprobación del aislamiento de la máquina y los ensayos de índice de polarización (IP).

\section{B. Técnicas de diagnóstico de faltas on line}

Este tipo de pruebas presentan una gran ventaja respecto a las técnicas de detección de faltas off line, ya que pueden realizarse en cualquier momento y en algunos casos de forma continua (monitorización). Las técnicas más extendidas son:

- Análisis de vibraciones.

- Análisis de ruidos.

- Termografía (análisis de la temperatura).

- Análisis eléctricos (corrientes, tensiones, potencia, par).

El ruido en las máquinas eléctricas está generado por las vibraciones de las mismas, por tanto existe una correlación entre el análisis espectral de las vibraciones y el análisis acústico, por lo que pueden detectarse las mismas faltas utilizando un método u otro. La detección del ruido presenta la ventaja de no necesitar contacto con la máquina (necesario en el análisis de vibraciones mediante acelerómetros), mientras que presenta el inconveniente de la interferencia con otras fuentes sonoras en un ambiente industrial [10].

El análisis termográfico se basa en que la mayoría de los componentes de un sistema muestran un incremento de temperatura en caso de mal funcionamiento. Por ejemplo, el incremento de temperatura en un circuito eléctrico podría deberse a una mala conexión y en el caso de equipos rotativos a problemas con un rodamiento. Observando el comportamiento térmico de los componentes pueden detectarse defectos y evaluar su seriedad. Para el análisis se utiliza una cámara termográfica, que mide la emisión natural de radiación infrarroja procedente de un objeto y genera una imagen térmica.

Los análisis eléctricos se centran principalmente en el estudio de las corrientes del estator, que presentan contenidos armónicos diferentes según el tipo de avería.

Todas estas técnicas de detección y diagnóstico de faltas están bien documentadas para el caso del motor de inducción, sin embargo, en los accionamientos SRM el análisis de faltas empezó a estudiarse a partir del trabajo de Stephens en 1991 [11], posteriormente numerosos autores han hecho importantes contribuciones al tema [12-18].

\section{Propuesta De Una Guía De Mantenimiento Predictivo De ACCIONAMIENTOS CON SRM}

\section{A. Metodología propuesta}

Se presenta un plan de mantenimiento predictivo para accionamientos SRM centrado en las técnicas de diagnóstico on line, aunque también se realizaran, en los periodos de parada programada, comprobaciones del estado de aislamiento (medida de aislamiento y del índice de polarización). Es importante señalar que, en los análisis de mantenimiento predictivo, no es tan importante la exactitud del valor de la medida de una variable como el cambio que se ha producido desde la última inspección realizada [19].

En la Fig. 3 se muestra el diagrama de bloques de la de implementación del sistema de mantenimiento predictivo propuesto para accionamientos SRM. En primer lugar deben seleccionarse las máquinas a analizar. A continuación debe elegirse la técnica o técnicas óptimas para la detección, para seguidamente crear, al inicio del funcionamiento del equipo cuando éste funcione en perfecto estado o después de una reparación o corrección de faltas, las mediciones de referencia, 
así como determinar un punto de alarma que indique cuándo la maquina empieza a presentar problemas. Este punto de alarma debe fijarse teniendo en cuenta el tiempo de reacción del equipo de mantenimiento para proceder a la corrección de la falta. Una vez realizado el proceso descrito, se pasa a la toma de medidas de cada una de las técnicas seleccionadas, fijando un periodo de medida que dependerá de la gravedad de la falta y de las características de la máquina. Si la medida es correcta, comparándola con la referencia fijada, se recopilaran los datos y se analizaran las tendencias, es decir se preverá en cuánto tiempo se alcanzará el punto de alarma fijado. En el caso de que la medida alcance el punto de alarma, se comparará la medida con los patrones de falta que se han obtenido, determinando el tipo de falta que se ha producido y realizaremos la corrección de la falta. Una vez corregida volveremos a determinar la medida de referencia para volver a comenzar un nuevo ciclo.

Aunque en el estudio presentado la máquina seleccionada es el accionamiento SRM cuyas características se muestran en el apéndice con control de histéresis a bajas velocidades y control de pulso único para elevadas velocidades, se han realizado exhaustivas pruebas experimentales sobre otros accionamientos SRM de distinto tamaño [20] y de diferente configuración [21], en los que se han utilizado las diferentes técnicas de detección indicadas en la sección III, llegándose a la conclusión de que las técnicas más adecuadas para el caso de los accionamiento SRM son: el análisis de la corriente y el análisis espectral de la aceleración de la vibración.

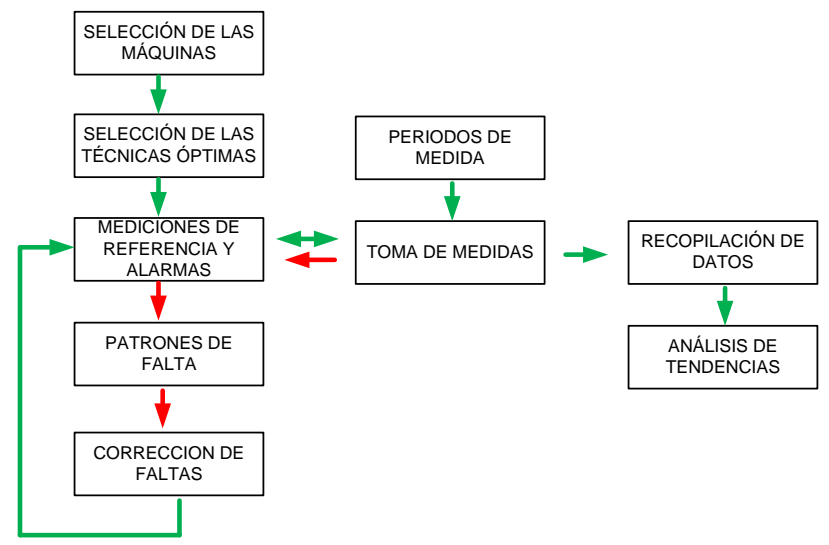

Fig. 3. Diagrama de implantación del sistema de mantenimiento predictivo propuesto para SRM.

\section{B. Determinación de las medidas de referencia}

En lo referente a las corrientes, se muestran en la Fig. 4 las corrientes de las cuatro fases funcionando con control de histéresis. En la Fig. 5 se pueden observar las corrientes de fase en funcionamiento con control de pulso único; obsérvese en este caso que existen algunas pequeñas variaciones de la corriente en cada una de las fases debido a cuestiones constructivas del prototipo.

En cuanto a las vibraciones, se establecen las medidas de referencia en el análisis espectral de la aceleración de la vibración para el funcionamiento correcto, es decir, funcionando las 4 fases. En las Figs. 6 y 7 se muestra el espectro obtenido con el control de histéresis y con control de pulso único, respectivamente. En lo referente a las corrientes, se muestran en la Fig. 4 las corrientes de las cuatro fases funcionando con control de histéresis. En la Fig. 5 se pueden observar las corrientes de fase en funcionamiento con control de pulso único; señalar que en este caso existen algunas pequeñas variaciones de la corriente en cada una de las fases debido a cuestiones constructivas del prototipo.

En cuanto a las vibraciones, se establecen las medidas de referencia mediante el análisis espectral de la aceleración de la vibración en las correctas condiciones de operación, es decir, funcionando las 4 fases. En las Figs. 6 y 7 se muestran los espectros obtenidos con control de histéresis y con control de pulso único, respectivamente.

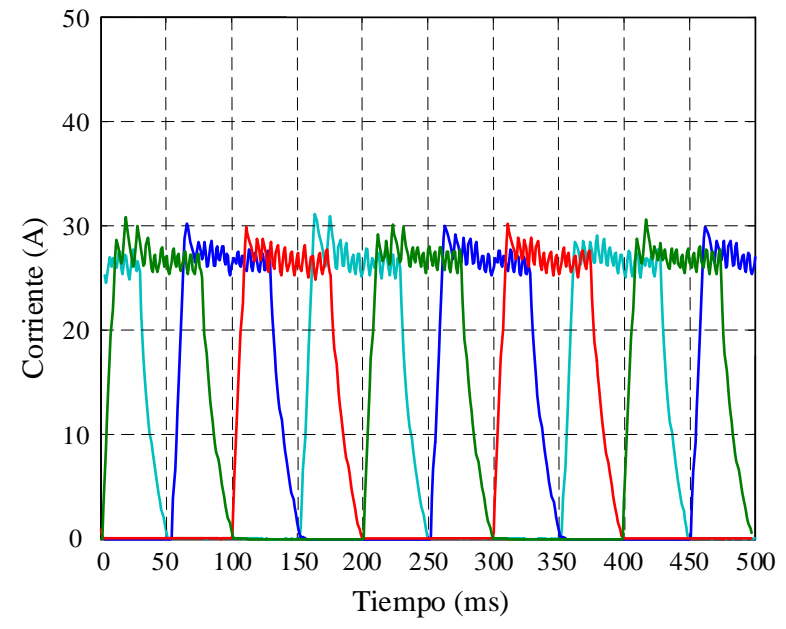

Fig. 4. Corrientes de fase (SRM 8/6 funcionando correctamente con control de histéresis). Condiciones de carga: 1 Nm, 1000 rpm.

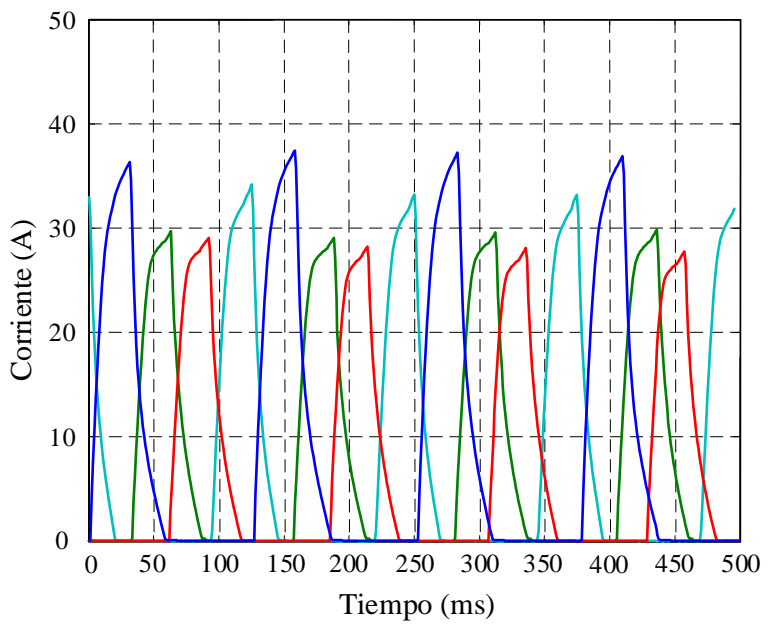

Fig. 5. Corrientes de fase (SRM 8/6 funcionando correctamente con control de pulso único). Condiciones de carga: $1 \mathrm{Nm}, 1580 \mathrm{rpm}$.

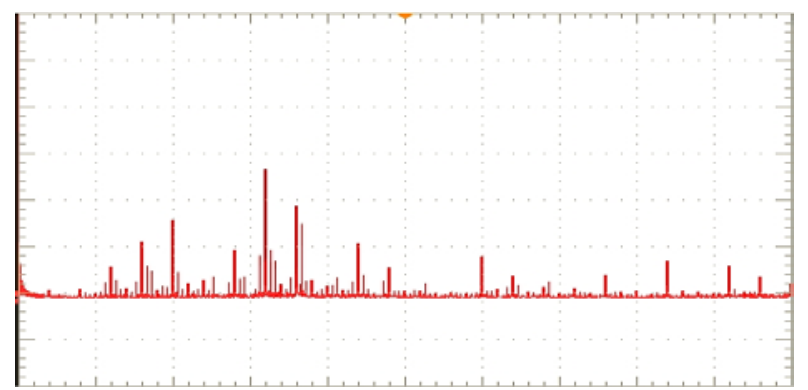

Fig. 6. Espectro de la aceleración de la vibración (SRM 8/6 funcionando correctamente con control de histéresis). Condiciones de carga: $1 \mathrm{Nm}, 1000$ rpm. Escalas: $1 \mathrm{~m} / \mathrm{s}^{2} / \operatorname{div}$ (ordenadas), $250 \mathrm{~Hz} / \operatorname{div}$ (abscisas). 


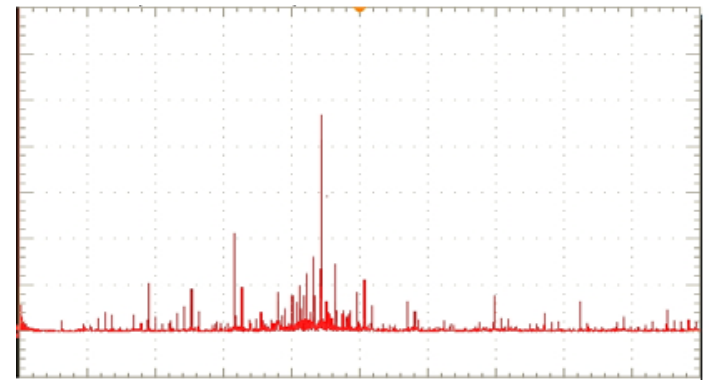

Fig. 7. Espectro de la aceleración de la vibración (SRM 8/6 funcionando correctamente con control de pulso único). Condiciones de carga: 1 Nm, 1580 $\mathrm{rpm}$. Escalas $1 \mathrm{~m} / \mathrm{s}^{2} / \mathrm{div}$ : (ordenadas), $250 \mathrm{~Hz} / \mathrm{div}$ (abscisas).

\section{Determinación de los patrones de falta}

Una vez obtenidas las medidas de referencia, identificaremos, en el caso que se produzca una variación de la medida obtenida con respecto a los valores de referencia, cuál es la falta que nos ha provocado dicha variación. Comparando dicha medida de referencia con las medidas realizadas cuando se ha producido una falta, estableceremos como patrón de falta aquella medida que nos proporcione una mayor información sobre la misma.

Analizaremos dos tipos de faltas: fase abierta y cortocircuito entre espiras en la bobina de un polo del estator.

- Fase abierta.

En la Fig. 8 se muestran las corrientes de fase funcionando el motor únicamente con tres fases y con control de histéresis. Observamos que la fase dañada deja de consumir corriente y, comparándola con la medida de referencia, podemos detectar el problema. Cabe destacar que las fases sanas no sufren alteraciones, lo que corrobora la independencia de fases del accionamiento. Evidentemente, para poder mantener las mismas condiciones de par, el consumo de corriente en las fases sanas ha aumentado respecto del funcionamiento correcto de la Fig. 4.

A continuación se ha realizado el mismo proceso para el control con pulso único. Se observa, Fig. 9, que la fase dañada deja de consumir corriente y aumenta el consumo en las fases sanas.

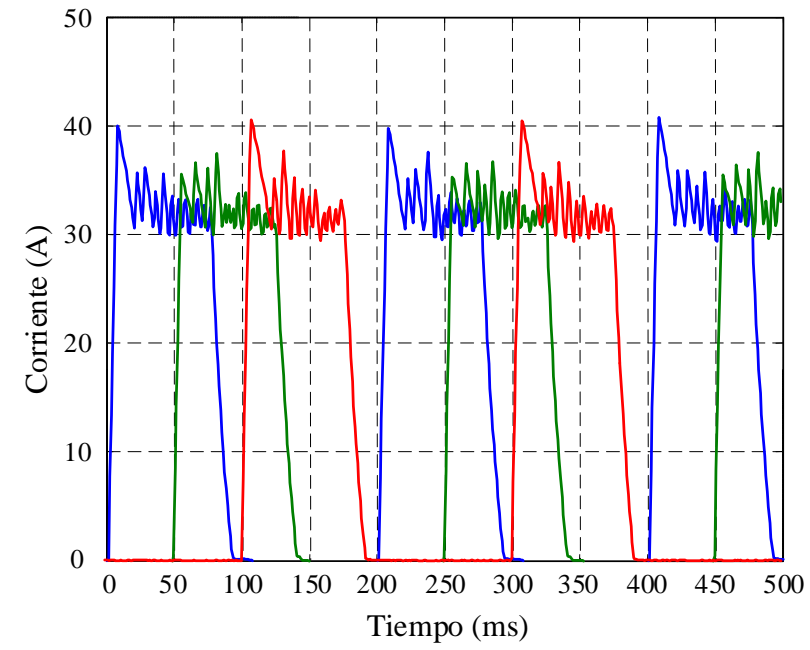

Fig. 8. Corrientes de fase (SRM 8/6 funcionando únicamente con 3 fases con control de histéresis). Condiciones de carga: 1 Nm, 1000 rpm.

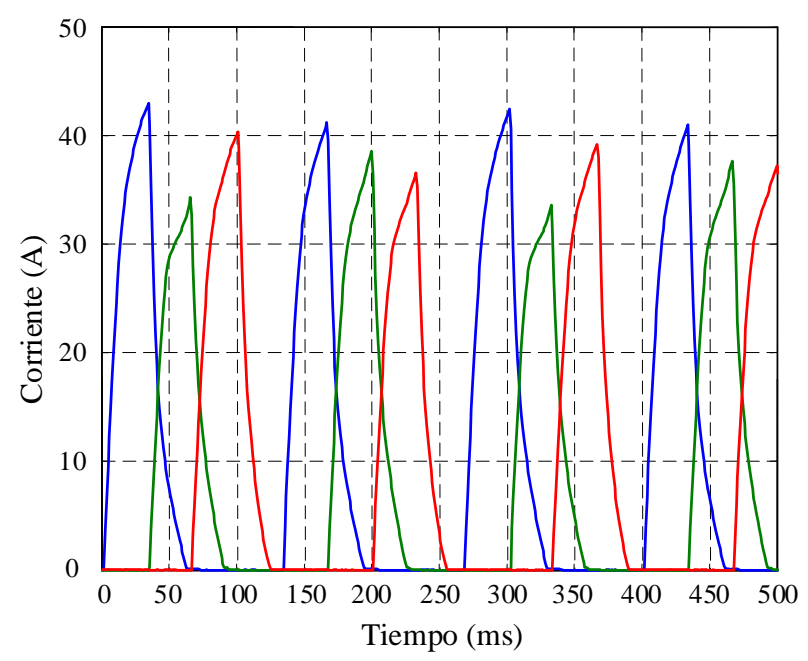

Fig. 9. Corrientes de fase (SRM 8/6 funcionando únicamente con 3 fases con control de pulso único). Condiciones de carga: 0,85 Nm, 1500 rpm.

En la Fig. 10 se muestra el espectro de la aceleración de la vibración con control de histéresis, comparada con la de referencia de la Fig. 6. En la Fig. 11 se comparan los espectros con control de pulso único. En ambos casos la diferencia entre el espectro de funcionamiento correcto y con el de fase abierta no es significativo y, por tanto, esta técnica no nos permite apreciar la falta tan claramente como en el caso del análisis de corrientes. Podemos concluir que en el caso de la falta de fase abierta, tanto con control de histéresis como de pulso único, la mejor técnica es el análisis de las corrientes de fase en detrimento del análisis espectral de la aceleración de la vibración.

- Cortocircuito entre espiras.

Se han simulado mediante las diferentes tomas accesibles del motor (ver apéndice) diferentes cortocircuitos entre espiras. En todos los casos se han incrementado los efectos apreciados al aumentar el número de espiras cortocircuitadas.

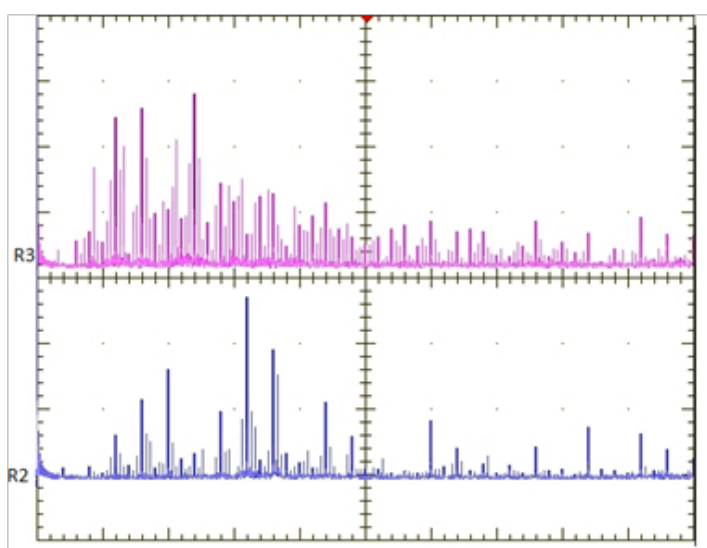

Fig. 10. Espectro de la aceleración de la vibración con control de histéresis (SRM 8/6, R2 funcionando correctamente, R3 funcionando con fase abierta). Condiciones de carga: $1 \mathrm{Nm}, 1000 \mathrm{rpm}$. Escalas: $1 \mathrm{~m} / \mathrm{s}^{2} / \mathrm{div}$ (ordenadas), 250 $\mathrm{Hz} / \operatorname{div}$ (abscisas). 


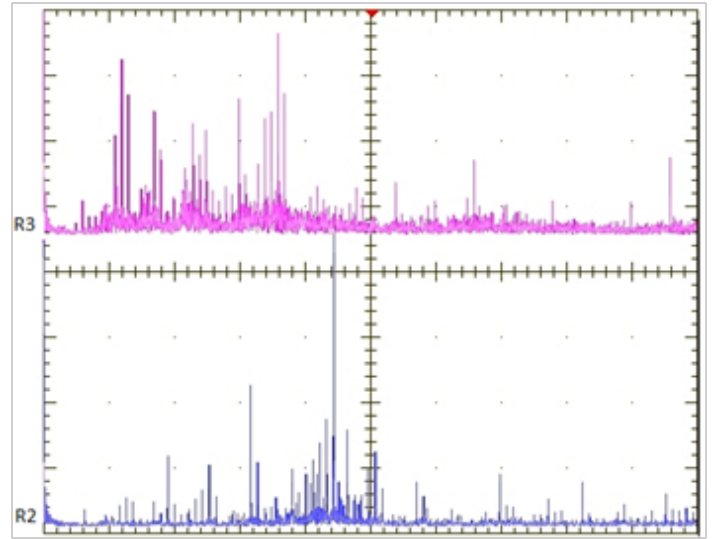

Fig. 11. Espectro de la aceleración de la vibración con control de pulso único (SRM 8/6, R2 funcionando correctamente, R3 funcionando con fase abierta). Condiciones de carga: 0,85 Nm, $1500 \mathrm{rpm}$. Escalas: $1 \mathrm{~m} / \mathrm{s}^{2} / \mathrm{div}$ (ordenadas), $250 \mathrm{~Hz} / \operatorname{div}$ (abscisas).

En la Fig. 12 se presentan las corrientes aplicando el control de histéresis. Para la misma carga y la misma velocidad de la medida de referencia, podemos observar que la fase cortocircuitada (fase D) presenta un pequeño incremento de corriente con respecto a las fases sanas, aunque no podemos decir que sea perceptible. Por tanto, no podemos identificar el fallo mediante este análisis, debido al control de corriente aplicado sobre el motor ya que no permite un incremento de la corriente superior al prefijado por el control.

En la Fig. 13 se muestran las corrientes con control de pulso único. Para este tipo de funcionamiento, la detección de la falta de cortocircuito entre espiras es posible mediante el análisis de corriente de las fases ya que, al no existir control de corriente, ésta se incrementa de forma proporcional a la cantidad de espiras cortocircuitadas. Las fases sanas no sufren alteraciones y se mantienen con los mismos valores que en el funcionamiento sin falta.

Por tanto, podemos decir que la técnica del análisis de corrientes para la detección de cortocircuitos entre espiras de una bobina será válida únicamente si el motor funciona con control de pulso único.

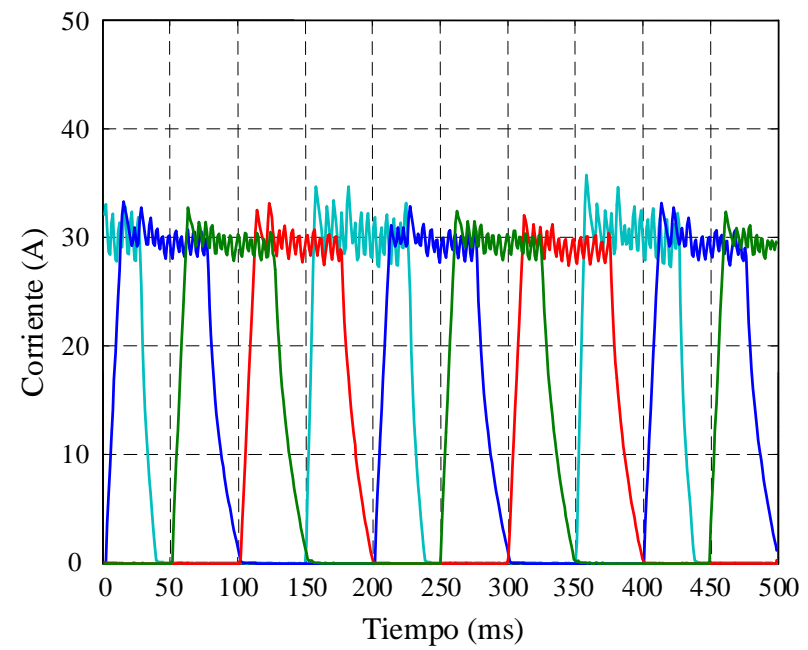

Fig. 12. Corrientes de fase (SRM 8/6 funcionando con cortocircuito de 16 espiras y control de histéresis). Condiciones de carga: 1 Nm, 1000 rpm.

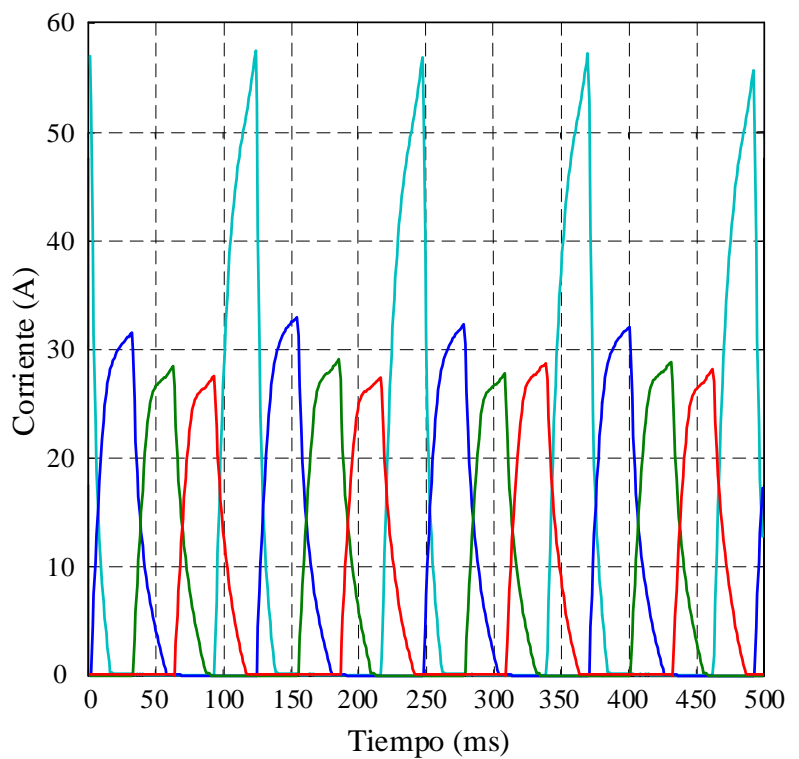

Fig. 13. Corrientes de fase (SRM 8/6 funcionando con cortocircuito de 16 espiras y control de pulso único). Condiciones de carga: 1 Nm, 1640 rpm.

A continuación analizaremos las vibraciones, comparando los resultados con el funcionamiento sin falta. Observamos en la Fig. 14 el espectro de la aceleración de la vibración con control de corriente. Como podemos ver se produce un incremento de la amplitud de la vibración y de la frecuencia fundamental, que varía con la velocidad según la ecuación (2).

$\mathrm{f}=\mathrm{k} \mathrm{N}_{\mathrm{r}} \frac{\mathrm{N}}{60}$

Dónde:

$\mathrm{k}=$ para todo número entero $\geq 1$.

$\mathrm{f}=$ frecuencia $(\mathrm{Hz})$.

$\mathrm{N}_{\mathrm{r}}=$ número de polos del rotor.

$\mathrm{N}=$ velocidad (rpm).

En la Fig. 15 se muestra el análisis espectral de la aceleración de la vibración trabajando el motor a pulso único. Comparando con la medida de referencia sin falta, podemos observar claramente el incremento de la amplitud de la aceleración de la vibración en el caso de cortocircuitos entre espiras, lo cual nos permite la detección de este tipo de falta para el funcionamiento a pulso único.

\section{Técnicas de diagnóstico a utilizar en función del tipo de} falta y según el tipo de control aplicado.

A partir del estudio experimental realizado por los autores, tanto en el motor SRM utilizado en este artículo como en otros motores ensayados, se puede afirmar que la técnica de diagnóstico más adecuada para la detección de faltas depende, además del tipo de falta, del tipo de control utilizado. En concreto, para los casos estudiados de faltas de fase abierta y de cortocircuito entre espiras, las mejores alternativas se recogen en la Tabla II. 


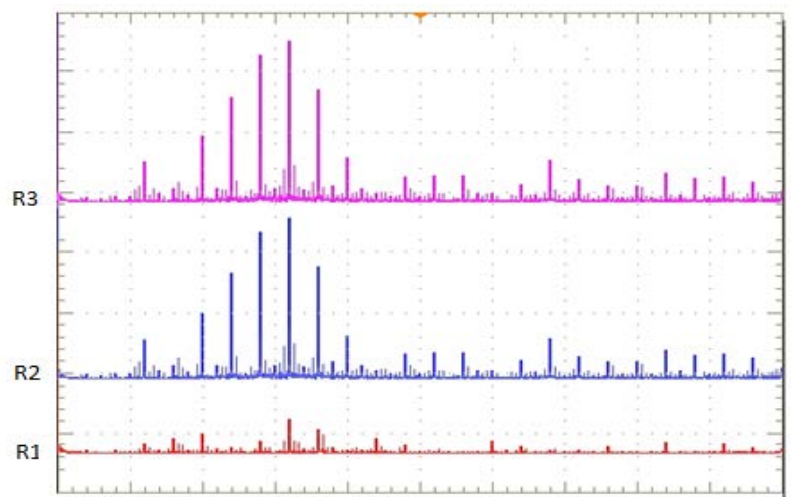

Fig. 14. Espectro de la aceleración de la vibración con control de-histéresis (SRM 8/6, R1 funcionando correctamente R2 funcionando con cortocircuito de 24 espiras, R3 funcionando con cortocircuito de 32 espiras). Condiciones de carga: $1 \mathrm{Nm}, 1000 \mathrm{rpm}$. Escalas: $5 \mathrm{~m} / \mathrm{s}^{2} / \mathrm{div}$ (ordenadas), $250 \mathrm{~Hz} / \mathrm{div}$ (abscisas).

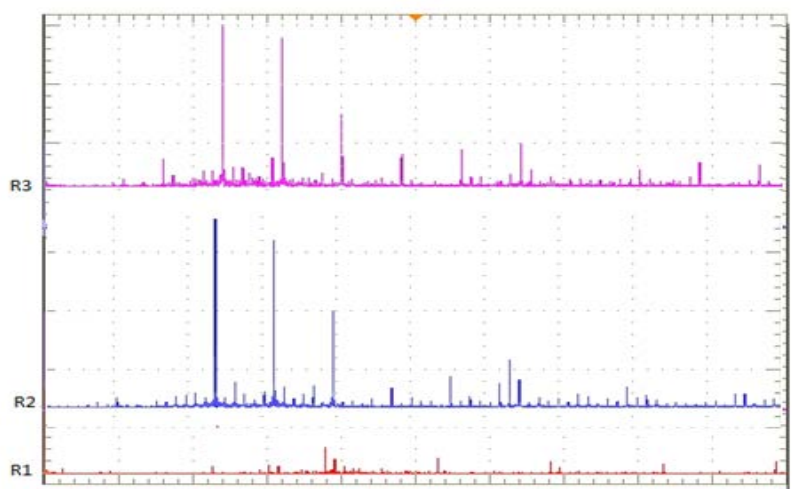

Fig. 15. Espectro de la aceleración de la vibración con control de pulso único (SRM 8/6, R1 funcionando correctamente R2 funcionando con cortocircuito de 24 espiras, R3 funcionando con cortocircuito de 32 espiras). Condiciones de carga: 0,5 Nm, $1900 \mathrm{rpm}$. Escalas: $10 \mathrm{~m} / \mathrm{s}^{2} / \mathrm{div}$ (ordenadas), $250 \mathrm{~Hz} / \mathrm{div}$ (abscisas).

TABLA II

TÉCNICAS A UTILIZAR EN LA DETECCIÓN DE FALTAS EN EL SRM

\begin{tabular}{ccccc}
\hline \hline & \multicolumn{2}{c}{$\begin{array}{c}\text { Detección con control } \\
\text { de histéresis }\end{array}$} & \multicolumn{2}{c}{$\begin{array}{c}\text { Detección con control de } \\
\text { pulso único }\end{array}$} \\
\hline Falta & $\begin{array}{c}\text { Análisis } \\
\text { corriente }\end{array}$ & $\begin{array}{c}\text { Análisis } \\
\text { vibraciones }\end{array}$ & $\begin{array}{c}\text { Análisis } \\
\text { corriente }\end{array}$ & $\begin{array}{c}\text { Análisis } \\
\text { vibraciones }\end{array}$ \\
Fase abierta & $\mathrm{Si}$ & $\mathrm{No}$ & $\mathrm{Si}$ & $\mathrm{No}$ \\
$\begin{array}{c}\text { Cortocircuito } \\
\text { entre espiras }\end{array}$ & $\mathrm{No}$ & $\mathrm{Si}$ & $\mathrm{Si}$ & $\mathrm{Si}$ \\
\hline \hline
\end{tabular}

\section{Conclusiones}

Los accionamientos SRM empiezan a encontrar su sitio en la industria, ante la próxima implementación de la Industria 4.0, y el mantenimiento predictivo tendrá un papel más destacado que el que tiene en la industria actual, por lo que es necesario implementar un plan de mantenimiento predictivo para este tipo emergente de accionamientos. Este artículo pretende hacer una aportación en este sentido, presentando un plan de mantenimiento predictivo para accionamientos SRM con diferentes estrategias de control, basados en técnicas on line.

Siguiendo la metodología del plan para el mantenimiento predictivo para los SRM propuesta en la sección IV, se obtuvieron las medidas de referencia para el funcionamiento correcto, mediante las medidas de la corriente y de las vibraciones con el motor en perfectas condiciones. Se establecieron los denominados patrones de faltas, a partir de las pruebas experimentales de falta sobre un accionamiento SRM. La comparación entre las medidas de referencia y los patrones de falta, tomadas en el accionamiento SRM en intervalos programados, permitieron determinar si hay presencia de una falta.

Se analizaron exhaustivamente las faltas de fase abierta y de cortocircuito entre espiras de una bobina de una fase de un polo del estator. Se utilizaron las técnicas de diagnóstico que se consideraron más adecuadas para este tipo de accionamientos: análisis de corrientes y análisis espectral de la aceleración de las vibraciones, mostrándose en la Tabla II, cuál es la técnica más adecuada ante cada una de las faltas estudiadas y según el tipo de control con el que opera el motor.

\section{APÉNDICE}

El accionamiento utilizado para el estudio presentado consta de un SRM tetrafásico con 8 polos en el estator y 6 en el rotor (Fig. 16, características principales en la Tabla III), de un convertidor electrónico de potencia en puente asimétrico o clásico y de un encoder incremental como sensor de posición.

El motor se ha bobinado exprofeso con cierta asimetría entre sus fases y con diferentes tomas en la bobina de uno de los polos (ver Tabla III). Como se puede observar en la Fig. 17, mediante 4 interruptores se han emulado las siguientes faltas: abriendo el interruptor I1 se emula la falta de fase abierta; cerrando los interruptores I2, I3 e I4 se han emulado los cortocircuitos de 1/4, 1/2 y 3/4 de las espiras de un polo respectivamente.

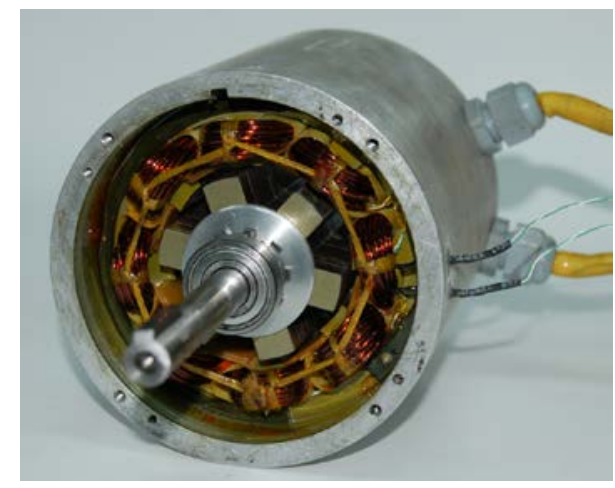

Fig. 16. Fotografía del SRM utilizado.

TABLA III CARACTERÍSTICAS DEL SRM UTILIZADO

\begin{tabular}{lc}
\hline \hline Potencia & $475 \mathrm{~W}$ \\
Tensión & $42 \mathrm{~V}$ \\
Velocidad & $1800 \mathrm{rpm}$ \\
Número de fases & 4 \\
Número de polos del estator & 8 \\
Número de polos del rotor & 6 \\
Resistencia de fase & $0,24 \Omega$ \\
Número de espiras por polo & 32 \\
Espiras seleccionables en la & \\
bobina con tomas & $8-16-24$ \\
\hline \hline
\end{tabular}




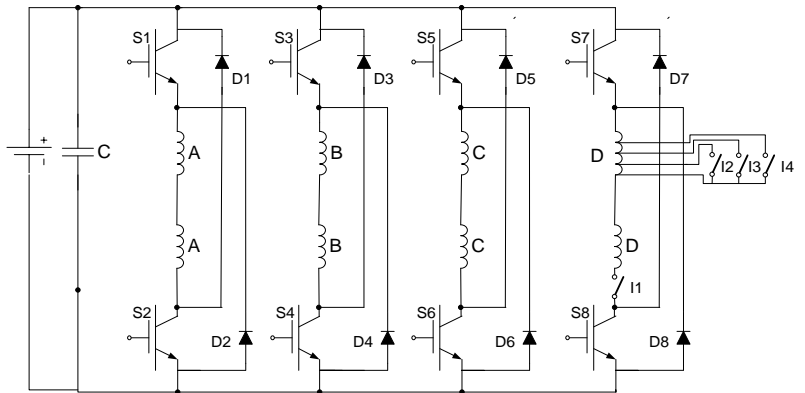

Fig. 17. Esquema de conexiones para la simulación de faltas.

\section{AGRADECIMIENTOS}

Esta investigación ha sido financiada por el Ministerio Español de Economía y Competitividad (DPI 2014-57086-R) y fondos FEDER.

\section{REFERENCIAS}

[1] B. Marr. "What Everyone Must Know About Industry 4.0", Forbes, June 2016.

[2] G. Changchao, H. Yihai, H. Xiao, C. Zhaoxiang, "Product Quality Oriented Predictive Maintenance Strategy for Manufacturing Systems", in Proc. Prognostics and System Health Management Conference, 2017, pp. 1-7.

[3] L. Bin, D.B. Durocher, P. Stemper, "Predictive Maintenance Techniques”, IEEE Industry Applications Magazine, vol. 15, no 6, pp. 52-60, 2009.

[4] A. Cachada, J. Barbosa, P. Leitão, C.A.S. Geraldes, L. Deusdado, J. Costa, C. Teixeira, J. Teixeira, A.H.J. Moreira, P.M. Moreira, L. Romero, "Maintenance 4.0: Intelligent and Predictive Maintenance System Architecture", in Proc. 2018 IEEE 23rd International Conference on Emerging Technologies and Factory Automation (ETFA), vol. 1, 2018, pp. 139-146.

[5] C.J. Verucchi, G.G. Acosta, "Fault Detection and Diagnosis Techniques in Induction Electrical Machines", IEEE Latin American Transactions, vol. 5, no 1, pp. 41-49, 2007.

[6] J.A. Antonino-Daviu, A. Quijano-López, M. Rubbiolo, V. ClimenteAlarcon, "Advanced Analysis of Motor Currents for the Diagnosis of the Rotor Condition in Electric Motors Operating in Mining Facilities", IEEE Transactions on Industry Applications, vol. 54, no 4, pp. 39343942, 2018

[7] "Switched reluctance motors market to 2021 - Global Analysis and Forecasts by Types, Technologies Applications and End-User Verticals". Wise Guy Reports.

[8] M. Ruba, C. Oprea, L. Szabo, "Comparative Study on Switched Reluctance Machine Based Fault-Tolerant Electrical Drive Systems”, in Proc. Electric Machines and Drives Conference, IEMDC 2009, pp. 987992.

[9] R. Terec, I. Bentia, M. Ruba, L. Szabo, P. Rafajdus, "Effects of Winding Faults on the Switched Reluctance Machine's Working Performances", in Proc. 3rd IEEE International Symposium on Logistics and Industrial Informatics, August 25-27, 2011, Budapest (Hungary).

[10] M.N. Anwar, I. Husain, S. Mir, T. Sebastian, "Evaluation of Acoustic Noise and Mode Frequencies with Design Variations of Switched Reluctance Machines", IEEE Transactions on Industry Applications, vol. 39, no 3, pp. 695-703, May-June 2003.

[11] Stephens, C.M., "Fault Detection and Management System for FaultTolerant Switched Reluctance Motor Drives", IEEE Transactions on Industry Applications, vol. 27, nº 6, pp. 1098-1102, Nov-Dec 1991.

[12] C. Hao, Z. Chao, "Analysis of Electrical Faults for Switched Reluctance Motor Drives", in Proc. 3rd World Congress Intelligent Control and Automation, vol. 1, no 28, pp. 666-669, June-July 2000.

[13] B. Lequesne, S. Gopalakrishnan, A.M. Omekanda, "Winding Short Circuits in the Switched Reluctance Drive", IEEE Transactions on Industry Applications, vol. 41, no 5, pp. 1178-1183, Sep/Oct 2005.

[14] S. Gopalakrishnan, A.M. Omekanda, B. Lequesne, "Classification and Remediation of Electrical Faults in the Switched Reluctance Drive", IEEE Transactions on Industry Applications, vol. 42, no 2, pp. 479-486, March/April 2006.
[15] G. Han, H. Chen, X. Shi, "Modelling, Diagnosis, and Tolerant Control of Phase-to-Phase Fault in Switched Reluctance Machine", IET Electric Power Applications, vol. 11, no 9, pp. 1527-1537, 2017.

[16] D.S.B. Fonseca, A.J. Marques, "On-line Stator Fault Diagnosis in Switched Reluctance Motors", in Proc. IEEE 11th International Symposium on Diagnostics for Electrical Machines, Power Electronics and Drives (SDEMPED), pp. 360-365, 2017.

[17] Y. Mollet, M. Sarrazin, H. Van der Auweraer, J. Gyselinck, "Investigation of the Influence of Open-phase Faults on Noise and Vibrations of Switched Reluctance Machines", in Proc. IEEE International Conference on Industrial Technology (ICIT), pp. 451-456, 2018.

[18] J. Shao, Z. Deng, Y. Gu, "Fault-Tolerant Control of Position Signals for Switched Reluctance Motor Drives", IEEE Transactions on Industry Applications, vol. 53, no 3, pp. 2959-2966, 2017.

[19] H. Opocenska, M. Hammer, "Use of Technical Diagnostics in Predictive Maintenance", in Proc. 17th International Conference on Mechatronics - Mechatronika (ME), pp. 1-6, 2016.

[20] M. Gomila, J.A. Sánchez, P. Andrada, B. Blanqué, E. Martínez, J.I. Perat, M. Torrent, "Accionamientos de Reluctancia Autoconmutados con Control de Par para Dirección Asistida Eléctrica”, Dyna, vol. 91, no 3, pp. 289-295, Mayo-Junio 2016.

[21] P. Andrada, E. Martínez, B. Blanqué, M. Torrent, J.I. Perat, J.A. Sánchez, "New Axial-Flux Switched Reluctance Motor for E-scooter", in Proc. ESARS ITEC, Toulouse, 2-4 November 2016.

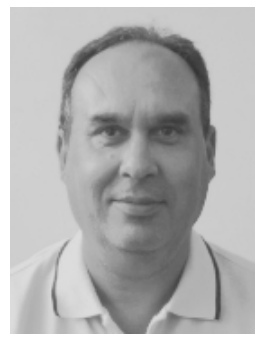

J A. Sánchez nació en Vilanova i la Geltrú, Barcelona, España, en 1953. Doctor por la Universitat Politècnica de Catalunya (UPC), Barcelona, en 2018. Es actualmente Profesor del Departamento de Ingeniería Eléctrica, en la Escola Politècnica Superior d'Enginyeria de Vilanova i la Geltrú, UPC.

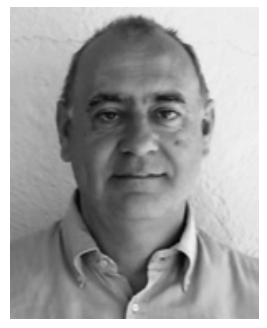

P. Andrada (M’91) nació en Barcelona, España, en 1957. Ingeniero Industrial y Doctor Ingeniero Industrial por la Universitat Politècnica de Catalunya (UPC), Barcelona, en 1980 y 1990, respectivamente. Desde 1980 trabaja en el Departamento de Ingeniería Eléctrica de la UPC, donde actualmente es Profesor en la Escola Politècnica Superior d'Enginyeria de Vilanova i la Geltrú, UPC.

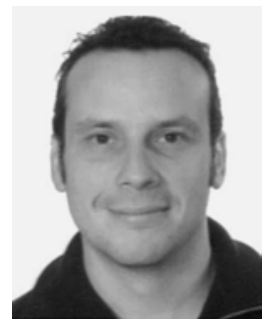

B. Blanqué nació en Reus, Tarragona, España, en 1970. Ingeniero de Telecomunicaciones y Doctor por la Universitat Politècnica de Catalunya (UPC), Barcelona, en, 1999 y 2007, respectivamente. Desde 1996, trabaja en el Departamento de Ingeniería Eléctrica de la UPC, actualmente es Profesor en la Escola Politècnica Superior d'Enginyeria de Vilanova i la Geltrú, UPC.

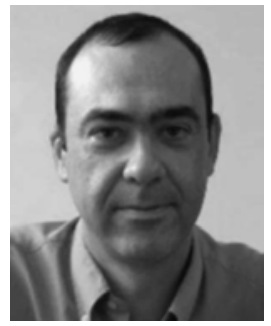

M. Torrent nació en Menàrguens, Lleida, España, en 1965. Doctor Ingeniero Industrial por la Universitat Politècnica de Catalunya (UPC), Barcelona, en 2002. Es actualmente Profesor del Departamento de Ingeniería Eléctrica, en la Escola Politècnica Superior d'Enginyeria de Vilanova i la Geltrú, UPC. 